\title{
Group classification of a class of generalized nonlinear Kolmogorov equations and exact solutions
}

\author{
I. Rassokha, ${ }^{1, a)}$ M. Serov, ${ }^{1, b)}$ S. Spichak, ${ }^{2, \text { c) }}$ and V. Stogniy ${ }^{3, d)}$ \\ 1) Poltava National Technical Yuri Kondratyuk University, 24 Pershotravnevyi Ave., \\ 36011 Poltava, Ukraine \\ 2) Institute of Mathematics, National Academy of Sciences of Ukraine, \\ 3 Tereshchenkivs'ka Str., 01004 Kyiv-4, Ukraine \\ 3) National Technical University of Ukraine "Igor Sikorsky \\ Kyiv Polytechnic Institute”, 37 Peremohy Ave., 03056 Kyiv, \\ Ukraine
}

(Dated: 24 October 2018)

A class of generalized nonlinear Kolmogorov equations is investigated. We present the group classification of Lie symmetries of the class with respect to the group of equivalence transformations. We find a number of exact solutions of nonlinear Kolmogorov equations which has the maximal symmetry properties.

PACS numbers: 02.20.Sv, 02.30.Jr, 92.60.Ek, 94.10.Lf

Keywords: generalized nonlinear Kolmogorov equation, group classification, exact solution

\footnotetext{
a) Electronic mail: innaolha@gmail.com

b) Electronic mail: mserov4@gmail.com

c) Electronic mail: spichak@imath.kiev.ua

d) Electronic mail: stogniyvaleriy@gmail.com
} 


\section{INTRODUCTION}

In this article, we study the class of the generalized nonlinear Kolmogorov type equations:

$$
u_{t}-u_{x x}+f^{1}(u) u_{y}=f^{2}(u)
$$

where $u=u(t, x), u_{t}=\frac{\partial u}{\partial t}, u_{x}=\frac{\partial u}{\partial x}, u_{x}=\frac{\partial u}{\partial x}, u_{x x}=\frac{\partial^{2} u}{\partial x^{2}} ; f^{1}(u), f^{2}(u)$ are arbitrary smooth functions of their variables, and $f^{1} \neq$ const. If $f^{1}=$ const then equation (1) can be reduced to a spatially one-dimensional nonlinear one $\left(f^{1}=0\right)$ by corresponding Galilean transformation. The group classification of such equations was carried out, in particular, in Ref. 6 .

Equation (1) occurs in problems of financial mathematics and in studying of the physical nonlinear phenomena such as the combined effects of diffusion and convection of matter ${ }^{19}$.

Wide application of equation (1) causes an undeniable interest in obtaining solutions. One of the most powerful methods for constructing exact solutions of nonlinear PDEs is the classical Lie method ${ }^{2,16,18}$, and its various generalizations and modifications ${ }^{11}$.

A common method for solving nonlinear differential equations is the Lie method, which is based on the principle of symmetry. S. Lie was the first who used an invariance algebra of differential equations for symmetry reduction and for finding exact solutions. The theoretical-group methods allow you to integrate differential equations, which have nontrivial invariance group. That's why the urgent task is a complete group classification of differential equations with arbitrary functions that allows to select the equations with broad symmetry properties from the given class of equations.

The interest in the group classification of equations exists for more than 50 years, since L.V. Ovsyannikov in his article ${ }^{17}$ has made the current formulation of the problem of group classification of differential equations, proposed a method for its solution and implemented a group classification of nonlinear heat equation. Detailed review of publications of group classification of differential equations until the mid 90-ies of the last century is given in Ref. 10. Group classification of evolution equations in spaces of dimension higher than the two-dimensional space-time is considered in Refs. 5, 7-9, 15, 24-26.

The problem of group classification of equation (1) for the case $f^{2}(u)=0$ was carried out in Refs. 4 and 8 relatively to an arbitrary function $f^{1}(u)$, and, in particular, shows that the 
nonlinear Kolmogorov equation

$$
u_{t}-u_{x x}-u u_{y}=0
$$

admits 6-parameter group of local transformations and using the method of symmetry reduction, built exact solutions of that equation.

Thus, it becomes apparent urgency of the task complete group classification of equation (1) with respect to arbitrary functions and which was resolved by us.

\section{THE BASIC ALGEBRA $A^{\text {ker }}$}

Consider a one-parameter Lie group of local transformations in a space of variables $(t, x, y, u)$ with an infinitesimal operator

$$
G=\xi^{0}(t, x, y, u) \partial_{t}+\xi^{1}(t, x, y, u) \partial_{x}+\xi^{2}(t, x, y, u) \partial_{y}+\eta(t, x, y, u) \partial_{u}
$$

which keeps equations (1) invariant. The Lie criterion of infinitesimal invariance yields the following determining equations for functions $\xi^{0}, \xi^{1}, \xi^{2}, \eta$ and also for arbitrary elements $f^{1}(u)$ and $f^{2}(u)$ :

$$
\begin{gathered}
\xi_{x}^{0}=\xi_{u}^{0}=\xi_{u}^{1}=\xi_{x}^{2}=\xi_{u}^{2}=\eta_{u u}=0, \\
\xi_{t}^{0}-2 \xi_{x}^{1}+\xi_{y}^{0} f^{1}=0, \\
\xi_{t}^{1}-\xi_{x x}^{1}+\xi_{y}^{1} f^{1}+2 \eta_{u x}=0, \\
\eta \dot{f}^{1}=\left(\xi_{y}^{2}-2 \xi_{x}^{1}\right) f^{1}+\xi_{t}^{2}, \\
\eta \dot{f}^{2}=\left(\eta_{u}-2 \xi_{x}^{1}\right) f^{2}+\eta_{y} f^{1}+\eta_{t}-\eta_{x x},
\end{gathered}
$$

where the dot above the functions $f^{1}, f^{2}$ denote differentiation with respect to $u$.

Equations (3) do not contain arbitrary elements. Integration of them yields

$$
\xi^{0}=\xi^{0}(t, y), \quad \xi^{1}=\xi^{1}(t, x, y), \quad \xi^{2}=\xi^{2}(t, y), \quad \eta=\alpha(t, x, y) u+\beta(t, x, y)
$$

where $\alpha, \beta$ are arbitrary smooth functions.

Since the functions $\xi^{0}, \xi^{1}$ and $\alpha$ do not on variable $u$ and $f^{1} \neq$ const we obtain from (4), (5), (8)

$$
\xi_{y}^{0}=0, \quad \xi_{t}^{0}-2 \xi_{x}^{1}=0, \quad \xi_{y}^{1}=0, \quad \xi_{t}^{1}=-2 \alpha_{x}
$$


Since (8) the solutions of equations (9) have the form

$$
\xi^{0}=\gamma(t), \quad \xi^{1}=\frac{1}{2} \gamma^{\prime}(t) x+\delta(t), \quad \alpha=-\frac{1}{8} \gamma^{\prime \prime}(t) x^{2}-\frac{1}{2} \delta^{\prime}(t) x+\mu(t, y),
$$

where $\gamma, \delta, \mu$ are arbitrary smooth functions.

Taking into account (8), (10) and differentiating equation (6) on $x$ we have $\left(\alpha_{x} u+\beta_{x}\right) \dot{f}^{1}=0$, that is $\alpha_{x}=\beta_{x}=0$. Then relations (10) has the form

$$
\xi^{0}=2 c_{1} t+c_{0}, \quad \xi^{1}=c_{1} x+c_{2}, \quad \xi^{2}=\xi^{2}(t, y), \quad \eta=\alpha(t, y) u+\beta(t, y),
$$

where $c_{0}, c_{1}, c_{2}$ are constants. So, equations (6), (7) has the form

$$
\begin{gathered}
(\alpha u+\beta) \dot{f}^{1}=\left(\xi_{y}^{2}-2 c_{1}\right) f^{1}+\xi_{t}^{2} \\
(\alpha u+\beta) \dot{f}^{2}=\left(\alpha-2 c_{1}\right) f^{2}+\left(\alpha_{y} u+\beta_{y}\right) f^{1}+\alpha_{t} u+\beta_{t} .
\end{gathered}
$$

Splitting system (12), (13) with respect to the arbitrary elements and their non-vanishing derivatives gives the "trivial" solution (11)

$$
\xi^{0}=c_{0}, \quad \xi^{1}=c_{2}, \quad \xi^{2}=c_{3}, \quad \alpha=\beta=0,
$$

which corresponds to the coefficients of the operators (2) from the basic Lie algebra $A^{\text {ker }}$ of (1). As a result, the following theorem is true.

Theorem 1 For arbitrary functions $f^{1}$ and $f^{2}$ the Lie symmetry algebra of the equation (1) is $A^{\text {ker }}=\left\langle\partial_{t}, \partial_{x}, \partial_{y}\right\rangle$.

The problem of group classification consists in finding of all possible inequivalent cases when the solution of system (12), (13) leads to Lie algebra $A^{\text {max }}$, that satisfies the condition $A^{\text {ker }} \subset A^{\text {max }}$, i.e., to four- and higher-dimensional Lie algebra.

\section{GROUP OF EQUIVALENCE TRANSFORMATIONS}

The next step of the algorithm of group classification is finding equivalence transformations ${ }^{1,14,18}$ of class (1).

Theorem 2 An arbitrary nonlinear Kolmogorov type equation of the form (1) can be mapped to another equation of the same form

$$
\bar{u}_{\bar{t}}-\bar{u}_{\bar{x} \bar{x}}+F^{1}(\bar{u}) \bar{u}_{\bar{y}}=F^{2}(\bar{u})
$$


by the local transformations

$$
\bar{t}=T(t, x, y, u), \quad \bar{x}=X(t, x, y, u), \quad \bar{y}=Y(t, x, y, u), \quad \bar{u}=U(t, x, y, u),
$$

with the correctly-specified smooth functions $T, X, Y$ and $U$ and Jacobian $J(t, x, y, u)=$ $\frac{\partial(T, X, Y, U)}{\partial(t, x, y, u)} \neq 0$, if these functions are of the form

$$
\bar{t}=a_{1}^{2} t+a_{2}, \quad \bar{x}=a_{1} x+a_{3}, \quad \bar{y}=Y(t, y), \quad \bar{u}=\theta(t, y) u+\nu(t, y),
$$

and following equalities take place:

$$
\begin{gathered}
Y_{t}+Y_{y} f^{1}(u)=a_{1}^{2} F^{1}(\bar{u}), \\
\theta_{t} u+\nu_{t}+\left(\theta_{y} u+\nu_{y}\right) f^{1}(u)+\theta f^{2}(u)=a_{1}^{2} F^{2}(\bar{u}),
\end{gathered}
$$

where $a_{1}, a_{2}, a_{3}$ are real arbitrary constants, $a_{1} \neq 0, Y_{y} \neq 0, \theta(t, y) \neq 0$.

From (16)-(18) we obtain the set of equivalence transformations which is the local transformations group $G^{\text {equiv }}$ preserving the form equations (1).

Theorem 3 Group $G^{\text {equiv }}$ of the equation (1) contains the following continuous transformations:

$$
\begin{aligned}
& \bar{t}=a_{1}^{2} t+a_{2}, \bar{x}=a_{1} x+a_{3}, \bar{y}=a_{4} y+a_{5} t+a_{6}, \bar{u}=a_{7} u+a_{8}, \\
& F^{1}=\frac{a_{4}}{a_{1}^{2}} f^{1}+\frac{a_{5}}{a_{1}^{2}}, F^{2}=\frac{a_{7}}{a_{1}^{2}} f^{2},
\end{aligned}
$$

where $a_{i}, i \in\{1, \ldots, 8\}$ are arbitrary constants, $a_{1} a_{4} a_{7} \neq 0$.

Note that the transformations (19) reduce equations (1) to (15) for arbitrary functions $f^{1}, f^{2}$. For special view of these functions there are another (additional) equivalence transformations. These transformations will be further applied to unite the equations (1) with the same Lie symmetry.

\section{CLASSIFICATION OF LIE SYMMETRIES}

The next step of the algorithm of group classification is finding a complete set of inequivalent equations (1) with respect to the transformations from $G^{\text {equiv }}$ which are invariant under Lie algebra $A^{\text {max }} \supset A^{\text {ker }}\left(\operatorname{dim} A^{\text {max }}>\operatorname{dim} A^{\text {ker }}\right)$. Hereinafter, the notation $A^{\text {max }}$ implies the fulfillment of this condition. 


\section{Classification of functions $f^{1}$}

To construct all possible form of the functions $f^{1}$ for which corresponding set of solutions of system (12), (13) is wider than "trivial" solution (14) we analyze these equations along zero and non-zero values of the $\alpha$ and $\beta$.

Consider the simplest Case when for any solution (11) $\alpha=0, \quad \beta=0$, i.e., $\eta=0$. Then system $(12),(13)$ is reduced to the algebraic one with respect to $f^{1}$ and $f^{2}$

$$
\left(\xi_{y}^{2}-2 c_{1}\right) f^{1}+\xi_{t}^{2}=0, \quad c_{1} f^{2}=0 .
$$

One easily sees that Lie algebra $A^{\max }$ occurs only under the condition $c_{1} \neq 0$, otherwise the general solution of (20) leads to (14) (corresponding to the algebra $A^{\mathrm{ker}}$ ).

The condition $c_{1} \neq 0$ immediately gives $f^{2} \equiv 0$. Therefore, system (12), (13) with arbitrary functions $f^{1} \neq$ const and $f^{2} \equiv 0$ has the general solution

$$
\xi^{0}=2 c_{1} t+c_{0}, \quad \xi^{1}=c_{1} x+c_{2}, \xi^{2}=2 c_{1} y+c_{3},
$$

where $c_{i}(i \in\{0,1,2,3\})$ are arbitrary constants. The solution (21) leads to Lie algebra

$$
\left\langle\partial_{t}, \partial_{x}, \partial_{y}, 2 t \partial_{t}+x \partial_{x}+2 y \partial_{y}\right\rangle .
$$

Let us consider the condition $\alpha^{2}+\beta^{2} \neq 0$. Since the functions $\xi^{2}, \alpha$ and $\beta$ do not on variable $u$, one can obtain possible view the function $f^{1}$ from ODE (12).

Theorem 4 If an equation of the form (1) admits algebra $A^{\max }$ which includes infinitesimal operator (2) with $\eta \neq 0$ then the function $f^{1}$ is equivalents with respect to the transformations $G^{\text {equiv }}$ one of the form: $u^{k}(k \neq 0), e^{u}, \ln u$.

Proof. Case (1) $\alpha=0, \beta \neq 0$. Let us consider values $t, x, y$ as some parameters for equation (12). The general solution of equation (12) has the form

$$
\begin{aligned}
& f^{1}=C \exp \left(\frac{\xi_{y}^{2}-2 c_{1}}{\beta} u\right)-\frac{\xi_{t}^{2}}{\xi_{y}^{2}-2 c_{1}} \text { if } \xi_{y}^{2}-2 c_{1} \neq 0, \\
& \text { or } f^{1}=\frac{\xi_{y}^{2}}{\beta} u+C \quad \text { if } \quad \xi_{y}^{2}-2 c_{1}=0,
\end{aligned}
$$

where $C=C(t, x, y)$ is an arbitrary function. Because the function $f^{1}$ is dependent on $u$ only, it will be of the form

$$
f^{1}=k_{1} u+k_{2} \quad\left(k_{1} \neq 0\right), \quad \text { or } \quad f^{1}=k_{1} e^{k_{2} u}+k_{3} \quad\left(k_{1}, k_{2} \neq 0\right),
$$


TABLE I. Classification of functions $f^{1}$

\begin{tabular}{llll}
\hline \hline No. & $f^{1}(u)$ & $\begin{array}{l}\text { Equivalence } \\
\text { transformations }\end{array}$ & $F^{1}(\bar{u})$ \\
\hline 1 & $k_{1} e^{k_{2} u}+k_{3}$, & $\bar{t}=t, \bar{x}=x, \bar{u}=k_{2} u$ & $e^{\bar{u}}$ \\
& $k_{1} \neq 0, k_{2} \neq 0$ & $\bar{y}=\frac{1}{k_{1}} y-\frac{k_{3}}{k_{1}} t$, & \\
2 & $k_{1} \ln \left(u+k_{2}\right)+k_{3}$, & $\bar{t}=t, \bar{x}=x, \bar{u}=u+k_{2}$ & $\ln \bar{u}$ \\
& $k_{1} \neq 0$ & $\bar{y}=\frac{1}{k_{1}} y-\frac{k_{3}}{k_{1}} t$, & \\
3 & $\left(k_{1} u+k_{2}\right)^{m}+k_{3}$, & $\bar{t}=t, \bar{y}=y-k_{3} t$, & $\bar{u}^{m}, m \neq 0$ \\
& $m \neq 0, k_{1} \neq 0$ & $\bar{x}=x, \bar{u}=k_{1} u+k_{2}$ & \\
\hline \hline
\end{tabular}

where $k_{i}(i \in\{1,2,3\})$ are arbitrary constants.

Case (2) $\alpha \neq 0$. The general solution of equation (12) has the form

$$
\begin{aligned}
& f^{1}=C(\alpha u+\beta)^{\frac{\xi_{y}^{2}-2 c_{1}}{\alpha}}-\frac{\xi_{t}^{2}}{\xi_{y}^{2}-2 c_{1}} \text { if } \xi_{y}^{2}-2 c_{1} \neq 0, \\
& \text { or } \quad f^{1}=\frac{\xi_{t}^{2}}{\alpha} \ln |\alpha u+\beta|+C \quad \text { if } \quad \xi_{y}^{2}-2 c_{1}=0,
\end{aligned}
$$

where $C=C(t, x, y)$ are arbitrary function. As the function $f^{1}$ is dependent on $u$ only, it will be of the form

$$
f^{1}=\left(k_{1} u+k_{2}\right)^{m}+k_{3}, \quad \text { or } \quad f^{1}=k_{1} \ln \left(u+k_{2}\right)+k_{3} \quad\left(m, k_{1} \neq 0\right),
$$

where $m, k_{i}(i \in\{1,2,3\})$ are arbitrary constants.

Combining all obtained in the Cases 1, 2 forms of functions $f^{1}$ and using the set of equivalence transformations (19) we get simplified view $F^{1}$ of these functions (see Table I).

\section{Classification of functions $f^{2}$}

For each simplified functions $f^{1}$ from Table I we find all possible species of the function $f^{2}$ (the necessary conditions) under solving classifying equation (13). As before we impose the requirement that the equation has solutions different from "trivial" one (14) which correspond to the algebra $A^{\mathrm{ker}}$.

Case (1) $f^{1}=e^{u}$. Substituting function $f^{1}=e^{u}$ in equation (12) we obtain the relations $\alpha=0, \quad \beta=\xi_{y}^{2}-2 c_{1}, \quad \xi_{t}^{2}=0$. So from relations (11) we find that

$$
\xi^{0}=2 c_{1} t+c_{0}, \quad \xi^{1}=c_{1} x+c_{2}, \quad \xi^{2}=\xi^{2}(y), \quad \beta=\xi_{y}^{2}-2 c_{1} .
$$


Then classifying equation (13) has the form

$$
\left(\xi_{y}^{2}-2 c_{1}\right) \dot{f}^{2}=-2 c_{1} f^{2}+\xi_{y y}^{2} e^{u}
$$

1) Let $\xi_{y y}^{2} \neq 0$. Differentiating both sides of equation $(22)$ by $y$, we obtain that $\xi_{y y}^{2} \dot{f}^{2}=$ $\xi_{y y y}^{2} e^{u}$. Hence, the general solution has the form

$$
f^{2}=k_{1} e^{u}+k_{2}
$$

where $k_{1}$ and $k_{2}$ are arbitrary constants.

2) Let $\xi_{y y}^{2}=0$, i.e., $\xi^{2}=c_{3} y+c_{4}$. Then equation (22) has the form

$$
\left(c_{3}-2 c_{1}\right) \dot{f}^{2}=-2 c_{1} f^{2}
$$

If $c_{3}-2 c_{1}=0$ then $c_{1} \neq 0$ (otherwise Lie algebra coincides $A^{\text {ker }}$ ) and $f^{2}=0$, that falls into the previous Case (1) $\left(k_{1}=k_{2}=0\right)$. If $c_{3}-2 c_{1} \neq 0$ then general solution of equation $(24)$ has the form

$$
f^{2}=k_{1} e^{m u}
$$

where $k_{1}$ and $m$ are arbitrary constants.

Case (2) $f^{1}=\ln u$. Substituting function $f^{1}=\ln u$ in equation (12) we obtain the relations $\alpha=\xi_{t}^{2}, \quad \beta=0, \quad \xi_{y}^{2}=2 c_{1}$. So from relations (11) we find that

$$
\xi^{0}=2 c_{1} t+c_{0}, \quad \xi^{1}=c_{1} x+c_{2}, \quad \xi^{2}=2 c_{1} y+q(t), \quad \alpha=q^{\prime}(t) .
$$

Then classifying equation (13) has the form

$$
q^{\prime}(t) u \dot{f}^{2}=\left(q^{\prime}(t)-2 c_{1}\right) f^{2}+q^{\prime \prime}(t) u
$$

1) Let $q^{\prime \prime}(t) \neq 0$. Differentiating both sides of equation (26) by $t$ and $u$, we have $\ddot{f}^{2}=$ $q^{\prime \prime \prime}\left(q^{\prime \prime} u\right)^{-1}$. Hence, the general solution has the form $f^{2}=k_{1} u \ln u+k_{2} u+k_{3}$, where $k_{1}$, $k_{2}$ and $k_{3}$ are arbitrary constants. Substituting that solution into equation (26), we obtain that $k_{3}=0$. So,

$$
f^{2}=k_{1} u \ln u+k_{2} u \text {. }
$$

2) Let $q^{\prime \prime}(t)=0$. If $q^{\prime}(t) \neq 0$ then from (26) we have

$$
f^{2}=k_{1} u^{m}
$$


where $k_{1}$ are an arbitrary constant. If $q^{\prime}(t)=0$ then from $(26)$ we have $f^{2}=0$ (because $\left.c_{1} \neq 0\right)$ that falls into case $(28)$ when $k_{1}=0$.

Case (3) $f^{1}=u^{m}, m \neq 0,1$. Substituting function $f^{1}=u^{m}$ in equation (12) we obtain the relations $\beta=\xi_{t}^{2}=0, \quad m \alpha=\xi_{y}^{2}-2 c_{1}$. So from relations (11) we find that

$$
\xi^{0}=2 c_{1} t+c_{0}, \quad \xi^{1}=c_{1} x+c_{2}, \quad \xi^{2}=\xi^{2}(y), \quad \alpha=\frac{\xi_{y}^{2}-2 c_{1}}{m} .
$$

Then classifying equation (13) has the form

$$
\frac{\xi_{y}^{2}-2 c_{1}}{m} u \dot{f}^{2}=\left(\frac{\xi_{y}^{2}-2 c_{1}}{m}-2 c_{1}\right) f^{2}+\frac{\xi_{y y}^{2}}{m} u^{m+1} .
$$

1) Let $\xi_{y y}^{2} \neq 0$. Differentiating both sides of equation (29) by $y$, we obtain that

$$
u \dot{f}^{2}=f^{2}+\frac{\xi_{y y y}^{2}}{\xi_{y y}^{2}} u^{m+1}
$$

Then the general solution of equation (30) has the form

$$
f^{2}=k_{1} u^{m+1}+k_{2} u
$$

where $k_{1}$ and $k_{2}$ are arbitrary constants.

2) Let $\xi_{y y}^{2}=0$, i.e. $\xi^{2}=c_{3} y+c_{4}$. Then equation (29) has the form

$$
\frac{c_{3}-2 c_{1}}{m} u \dot{f}^{2}=\left(\frac{c_{3}-2 c_{1}}{m}-2 c_{1}\right) f^{2}
$$

If $c_{3}-2 c_{1}=0$ then $c_{1} \neq 0$ (otherwise Lie algebra coincides with $A^{\text {ker }}$ ) and $f^{2}=0$, that falls into the previous case (31). If $c_{3}-2 c_{1} \neq 0$ then general solution of equation (32) has the form

$$
f^{2}=k_{1} u^{n}
$$

Case (4) $f^{1}=u$. Substituting function $f^{1}=u$ in equation (12) and taking account (11) we find relations

$$
\xi^{0}=2 c_{1} t+c_{0}, \quad \xi^{1}=c_{1} x+c_{2}, \quad \xi^{2}=\xi^{2}(t, y), \quad \alpha=\xi_{y}^{2}-2 c_{1}, \quad \beta=\xi_{t}^{2}
$$

1) Let $\alpha=\beta=0$. In that case $c_{1} \neq 0$ (otherwise Lie algebra coincides with $A^{\text {ker }}$ ). Then it is following from (13) that $f^{2}=0$ what falls into the case (21). 
2) Let $\alpha=0, \beta \neq 0$. From (34) we obtain one of the possible general form of function $f^{2}$ for equation (13)

$$
\begin{gathered}
f^{2}=\frac{\beta_{y}}{2 \beta} u^{2}+\frac{\beta_{t}-\beta_{x x}}{\beta} u+C \quad\left(c_{1}=0\right), \\
f^{2}=C \exp \left(-\frac{2 c_{1}}{\beta} u\right)+\frac{\beta_{y}}{2 c_{1}} u+\frac{2 c_{1} \beta_{t}-\beta \beta_{y}}{4 c_{1}^{2}} \quad\left(c_{1} \neq 0\right),
\end{gathered}
$$

where $C(t, x, y)$ are an arbitrary function.

As the function $f^{2}$ is dependent on $u$ only, it will be of the form

$$
f^{2}=k_{1} u^{2}+k_{2} u+k_{3} \quad \text { or } \quad f^{2}=k_{1} \exp \left(k_{2} u\right)+k_{3} u+k_{4} \quad\left(k_{i} \neq 0, i \in\{1,2\}\right),
$$

where $k_{i}(i \in\{1,2,3,4\})$ are arbitrary constants.

If substitute $f^{2}=k_{1} u^{2}+k_{2} u+k_{3}$ when $k_{1} \neq 0$ into (13), then the system has the solution which leads to the algebra $A^{\mathrm{ker}}$. Also the function $f^{2}=k_{1} \exp \left(k_{2} u\right)+k_{3} u+k_{4}$ $\left(k_{i} \neq 0, i \in\{1,2\}\right), k_{3}^{2}+k_{4}^{2} \neq 0$ leads to the algebra $A^{\text {ker }}$ for equation (1). So in this case the possible form of the function under consideration has the view

$$
f^{2}=k_{1} u+k_{2}, \quad f^{2}=k_{1} \exp \left(k_{2} u\right),
$$

where $k_{i}(i \in\{1,2\})$ are arbitrary constants.

3) Let $\alpha \neq 0$. Similarly as in the case 1) the analysis of equation (13) leads to the following possible forms of the function $f^{2}$ :

$$
\begin{gathered}
f^{2}=k_{3} u^{i} \ln u+k_{2} u^{2}+k_{1} u+k_{0}, \quad i \in\{0,1,2\} \\
f^{2}=k_{3} u^{m}+k_{2} u^{2}+k_{1} u+k_{0}, \quad m \neq 0,1,2 ; \quad k_{3} \neq 0 .
\end{gathered}
$$

Analyzing of equation (13) one can obtain the following results. The general solution of that equation under functions (36) with $k_{3} \neq 0$ coincides with (14), what corresponds to the algebra $A^{\mathrm{ker}}$.

So, under $f^{1}=u$ one of the possible form of the function $f^{2}$ (up to redesignation of the constants $\left.k_{2}, k_{1}, k_{0}\right)$ is

$$
f^{2}=k_{1}\left(u+k_{2}\right)^{2}+k_{3}
$$

Further, if in function (37) $m \neq 3$ and $k_{2}^{2}+k_{1}^{2}+k_{0}^{2} \neq 0$ then the general solution of equation (13) has "trivial" view (14). Consequently, another of the possible form of the function $f^{2}$ is

$$
f^{2}=k_{3} u^{m}, \quad m \neq 0,1,2,3, \quad k_{3} \neq 0
$$


TABLE II. Classification of functions $f^{2}$

\begin{tabular}{llll}
\hline \hline No. & $f^{1}(u)$ & $f^{2}(u)$ & $F^{2}(\bar{u})$ \\
\hline 1 & $\forall$ & 0 & 0 \\
2 & $e^{u}$ & $k_{1} e^{u}+k_{2}, k_{1}, k_{2} \neq 0$ & $\varepsilon_{1} e^{\bar{u}}+\varepsilon_{2}$ \\
3 & $e^{u}$ & $k_{1} e^{m u}$ & $\tilde{\varepsilon}_{1} e^{m \bar{u}}$ \\
4 & $\ln u$ & $k_{1} u^{m}$ & $\tilde{\varepsilon}_{1} \bar{u}^{m}$ \\
5 & $\ln u$ & $k_{1} u \ln u+k_{2} u, k_{1} \neq 0$ & $\varepsilon_{1} \bar{u} \ln \bar{u}$ \\
6 & $u^{m}, m \neq 0,1$ & $k_{1} u^{n}$ & $\tilde{\varepsilon}_{1} \bar{u}^{n}$ \\
7 & $u^{m}, m \neq 0,1$ & $k_{1} u^{m+1}+k_{2} u, k_{1}, k_{2} \neq 0$ & $\varepsilon_{1} \bar{u}^{m+1}+\varepsilon_{2} \bar{u}$ \\
8 & $u$ & $k_{1} e^{k_{2} u}, k_{1}, k_{2} \neq 0$ & $\varepsilon_{1} e^{\bar{u}}$ \\
9 & $u$ & $k_{1} u^{m}, k_{1} \neq 0, m \neq 0,1,2,3$ & $\varepsilon_{1} \bar{u}^{m}$ \\
10 & $u$ & $k_{1}\left(u+k_{2}\right)^{3}, k_{1} \neq 0$ & $\varepsilon_{1} \bar{u}^{3}$ \\
11 & $u$ & $k_{1}\left(u+k_{2}\right)^{2}+k_{3}, k_{1} \neq 0$ & $\bar{u}^{2}+\tilde{\varepsilon}_{1}$ \\
12 & $u$ & $k_{1} u+k_{2}, k_{1} \neq 0$ & $\varepsilon_{1} \bar{u}$ \\
13 & $u$ & $k_{1}$ & $\tilde{\varepsilon}_{1}$ \\
\hline \hline
\end{tabular}

Here $\varepsilon_{i}= \pm 1, \tilde{\varepsilon}_{i} \in\{-1,0,1\}, i \in\{1,2\}$.

If in function (37) $m=3$ then corresponding "nontrivial" solution of (13) is only when

$$
f^{2}=k_{3} u^{3}+3 k_{3} k_{2} u^{2}+3 k_{3} k_{2}^{2} u+k_{3} k_{2}^{3}=k_{3}\left(u+k_{2}\right)^{3} .
$$

So, we combine:

functions $f^{2}(23)$, (25), corresponding the function $f^{1}=e^{u}$;

functions $f^{2}(27),(28)$, corresponding the function $f^{1}=\ln u$;

functions $f^{2}(31),(33)$, corresponding the function $f^{1}=u^{m}(m \neq 0,1)$;

functions $f^{2}(35)$, (38), (39), (40), corresponding the function $f^{1}=u$.

function $f^{2}=0$, corresponding an arbitrary function $f^{1}$ (see(20), (21)).

As result we have the following possible forms of the functions $f^{1}, f^{2}$ given in the Table II (columns two and tree).

Using the set of equivalence transformations (19) which do not change the form of the functions $f^{1}$ from Table I we get simplified view $F^{2}$ of the obtained functions $f^{2}$ (see Table II, column four). 


\section{Symmetry classification}

If an equation of the form (1) admits Lie algebra $A^{\max } \supset A^{\text {ker }}$, then it belongs to one of subclasses listed in the Table II. To get the standard group classification of the equation (1) now we need to find all possible Lie symmetries of each of those equations. For this we substitute each pair of the functions $f^{1}(u), f^{2}(u)=F^{2}(u)$ from the Table II (columns two and four) to equations (12), (13) under different values of $\varepsilon_{i}$, $\tilde{\varepsilon}$ and find general view of infinitesimal operator (2), (11).

As result we obtain the following theorem.

Theorem 5 Any equation of the form (1) which is invariant under Lie algebra $A^{\mathrm{max}} \supset A^{\mathrm{ker}}$ is mapped by an equivalence transformations group $G^{\text {equiv }}$ of the form (19) to one of the equations which are presented by functions $f^{1}$ and $f^{2}$ in Table III (columns two and three). The last column of the Table presents generators of an algebra $A^{\max }$ that do not belong to the algebra $A^{\mathrm{ker}}$.

\section{THE ADDITIONAL EQUIVALENCE TRANSFORMATIONS}

In this section we shall demonstrate the efficiency of using additional equivalence transformations for simplification of nonlinear Kolmogorov equations arising in Table III ${ }^{3,13,23,27}$. The ways of finding additional equivalence transformations is based on the fact that equivalent equations have the same dimensions of their invariance algebras $A^{\text {max }}$. To find additional equivalence transformations which reduced equation (1) to another equation of same form use conditions (16)-(18). From (17) it is easy to derive that locally equivalent equations from Table III must have same function $f^{1}(u)$. So, it is easy to go through all the pairs of equations in Table III, for which the dimensions of corresponding algebras and functions $f^{1}(u)$ are the same and verify their equivalence.

Let us give some examples how to find such pairs of equivalent equations.

\section{Six-dimensional invariance algebras}

1) Let us consider the cases 21 and 22 of the equations from Table III, which admit sixdimensional invariance algebras. Let us construct the additional equivalence transformations 
TABLE III. Group classification of equations (19) with respect to the $G^{\text {equiv }}$

\begin{tabular}{|c|c|c|c|}
\hline No. & $f^{1}(u)$ & $f^{2}(u)$ & Generators from $A^{\mathrm{max}} / A^{\mathrm{ker}}$ \\
\hline 1 & $\forall \neq$ const & 0 & $t \partial_{t}+\frac{1}{2} x \partial_{x}+y \partial_{y}$ \\
\hline 2 & $e^{u}$ & $\varepsilon_{1} e^{m u}, m \neq 0,1$ & $t \partial_{t}+\frac{1}{2} x \partial_{x}+\frac{m-1}{m} y \partial_{y}-\frac{1}{m} \partial_{u}$ \\
\hline 3 & $e^{u}$ & $\varepsilon_{1} e^{u}+\varepsilon_{2}$ & $e^{\varepsilon_{1} y}\left(\partial_{y}+\varepsilon_{1} \partial_{u}\right)$ \\
\hline 4 & $e^{u}$ & $\varepsilon_{1}$ & $y \partial_{y}+\partial_{u}$ \\
\hline 5 & $e^{u}$ & $\varepsilon_{1} e^{u}$ & $t \partial_{t}+\frac{1}{2} x \partial_{x}-\partial_{u}, \quad e^{\varepsilon_{1} y}\left(\partial_{y}+\varepsilon_{1} \partial_{u}\right)$ \\
\hline 6 & $e^{u}$ & 0 & $t \partial_{t}+\frac{1}{2} x \partial_{x}+y \partial_{y}, \quad y \partial_{y}+\partial_{u}$ \\
\hline 7 & $\ln u$ & $\varepsilon_{1} u^{m}, m \neq 1$ & $t \partial_{t}+\frac{1}{2} x \partial_{x}+\left(y-\frac{t}{m-1} \partial_{y}\right)-\frac{u}{m-1} \partial_{u}$ \\
\hline 8 & $\ln u$ & $\varepsilon_{1} u \ln u$ & $e^{\varepsilon_{1} t}\left(\partial_{y}+\varepsilon_{1} u \partial_{u}\right)$ \\
\hline 9 & $\ln u$ & $\varepsilon_{1} u$ & $t \partial_{t}+\frac{1}{2} x \partial_{x}+\left(y+\frac{\varepsilon_{1}}{2} t^{2} \partial_{y}\right)+\varepsilon_{1} t u \partial_{u}, \quad t \partial_{y}$ \\
\hline 10 & $\ln u$ & 0 & $t \partial_{t}+\frac{1}{2} x \partial_{x}+y \partial_{y}, \quad t \partial_{y}+u \partial_{u}$ \\
\hline 11 & $u^{m} m \neq 0,1$ & $\varepsilon_{1} u^{n} \neq m+1$ & $(n-1) t \partial_{t}+\frac{1}{2}(n-1) x \partial_{x}+(n-m-1) y \partial_{y}$ \\
\hline 12 & $u^{m} m \neq 0,1$ & $\varepsilon_{1} u^{m+1}+\varepsilon_{2} u$ & $e^{\varepsilon_{1} m y}\left(\partial_{y}+\varepsilon_{1} u \partial_{u}\right)$ \\
\hline 13 & $u^{m} m \neq 0,1$ & $\varepsilon_{1} u^{m+1}$ & $t \partial_{t}+\frac{1}{2} x \partial_{x}-\frac{1}{m} u \partial_{u}, \quad e^{\varepsilon_{1} m y}\left(\partial_{y}+\varepsilon_{1} u \partial_{u}\right)$ \\
\hline 14 & $u^{m} m \neq 0,1$ & 0 & $t \partial_{t}+\frac{1}{2} x \partial_{x}+y \partial_{y}, \quad y \partial_{y}+\frac{1}{m} u \partial_{u}$ \\
\hline 15 & $u$ & $\varepsilon_{1} e^{u}$ & $t \partial_{t}+\frac{1}{2} x \partial_{x}+(y-t) \partial_{y}-\partial_{u}$ \\
\hline 16 & $u$ & $\varepsilon_{1} u^{m} m \neq 0,1,2$ & $t \partial_{t}+\frac{1}{2} x \partial_{x}+\frac{m-2}{m-1} y \partial_{y}-\frac{u}{m-1} \partial_{u}$ \\
\hline 17 & $u$ & $u^{2}+1$ & $\begin{array}{l}e^{y}\left(\sin t \partial_{y}+(\cos t+u \sin t) \partial_{u}\right) \\
e^{y}\left(\cos t \partial_{y}-(\sin t-u \cos t) \partial_{u}\right)\end{array}$ \\
\hline 18 & $u$ & $u^{2}-1$ & $\begin{array}{l}e^{y}\left(\sinh t \partial_{y}+(\cosh t+u \sinh t) \partial_{u}\right) \\
e^{y}\left(\cosh t \partial_{y}+(\sinh t+u \cosh t) \partial_{u}\right)\end{array}$ \\
\hline 19 & $u$ & $\varepsilon_{1} u$ & $y \partial_{y}+u \partial_{u}, \quad e^{\varepsilon_{1} t}\left(\partial_{y}+\varepsilon_{1} \partial_{u}\right)$ \\
\hline 20 & $u$ & $u^{2}$ & $\begin{array}{l}t \partial_{t}+\frac{1}{2} x \partial_{x}-u \partial_{u}, \quad e^{y}\left(\partial_{y}+u \partial_{u}\right) \\
e^{y}\left(t \partial_{y}+(t u+1) \partial_{u}\right)\end{array}$ \\
\hline 21 & $u$ & $\varepsilon_{1}$ & $\begin{array}{l}t \partial_{t}+\frac{1}{2} x \partial_{x}+\varepsilon_{1} t^{2} \partial_{y}+\left(2 \varepsilon_{1} t-u\right) \partial_{u} \\
t \partial_{y}+\partial_{u}, \quad\left(y-\frac{1}{2} \varepsilon_{1} t^{2}\right) \partial_{y}+\left(u-\varepsilon_{1} t\right) \partial_{u}\end{array}$ \\
\hline 22 & $u$ & 0 & $t \partial_{t}+\frac{1}{2} x \partial_{x}+y \partial_{y}, \quad t \partial_{y}+\partial_{u}, \quad y \partial_{y}+u \partial_{u}$ \\
\hline
\end{tabular}

Here $\varepsilon_{i}= \pm 1, i \in\{1,2\}$. 
reducing equation

$$
u_{t}-u_{x x}+u u_{y}=\varepsilon_{1},
$$

to the equation

$$
u_{t}-u_{x x}+u u_{y}=0
$$

Taking into account (16) conditions (17) and (18) take the forms

$$
Y_{t}+Y_{y} u=a_{1}^{2}(\theta u+\nu), \quad \theta_{t} u+\nu_{t}+\left(\theta_{y} u+\nu_{y}\right) u+\theta \varepsilon_{1}=0
$$

Since the $\theta$ and $\nu$ do not depend on the variable $u$ we immediately arrive at

$$
Y_{t}=a_{1}^{2} \nu, \quad Y_{y}=a_{1}^{2} \theta, \quad \theta_{y}=0, \quad \theta_{t}+\nu_{y}=0, \quad \nu_{t}+\theta \varepsilon_{1}=0
$$

Solutions of that system have the form

$$
Y=C_{1} a_{1}^{2} y-\frac{C_{1} a_{1}^{2} \varepsilon_{1}}{2} t^{2}+C_{2} a_{1}^{2} t+C_{3}, \quad \theta=C_{1}, \quad \nu=-C_{1} \varepsilon_{1} t+C_{2},
$$

where $C_{1}, C_{2}, C_{3}$ are arbitrary constants, $C_{1} a_{1} \neq 0$.

Taking into account (43) we get relevant additional equivalence transformations

$$
\bar{t}=t, \quad \bar{x}=x, \quad \bar{y}=y-\frac{\varepsilon_{1}}{2} t^{2}, \quad \bar{u}=u-\varepsilon_{1} t,
$$

which reducing equation (41) to the equation (42) (omitting the top line for new variables).

2) Similarly, the case 20 of the equation from Table III

$$
u_{t}-u_{x x}+u u_{y}=u^{2}
$$

is reduced to the case 22 by the additional equivalence transformations

$$
\bar{t}=t, \quad \bar{x}=x, \quad \bar{y}=-e^{-y}, \quad \bar{u}=e^{-y} u .
$$

\section{Five-dimensional invariance algebras}

There are 9 equations admitting five-dimensional invariance algebra. It turns out that only five among them are not locally equivalent.

3) We consider the cases 18 and 19 of the equations from Table III, which admit fivedimensional invariance algebras. Let us construct the additional equivalence transformations reducing equation

$$
u_{t}-u_{x x}+u u_{y}=u^{2}-1
$$


to the equation

$$
u_{t}-u_{x x}+u u_{y}=u \text {. }
$$

The corresponding conditions (17) and (18)

$$
Y_{t}+Y_{y} u=a_{1}^{2}(\theta u+\nu), \quad \theta_{t} u+\nu_{t}+\left(\theta_{y} u+\nu_{y}\right) u+\theta\left(u^{2}-1\right)=a_{1}^{2}(\theta u+\nu)
$$

have a solution, which gives the following relevant additional equivalence transformations

$$
\bar{t}=2 t, \quad \bar{x}=\sqrt{2} x, \quad \bar{y}=-2 e^{t-y}, \quad \bar{u}=e^{t-y} u-e^{t-y} .
$$

These transformations reduces equation (45) to the equation (46).

4) Let us consider the cases 17 and 19 of the equations from Table III. It turns out that these equations are not equivalent. Assume the contrary. Then, similarly to the previous case 3 we obtain the following equation (17) and (18) to search for equivalence transformations:

$$
Y_{t}+Y_{y} u=a_{1}^{2}(\theta u+\nu), \quad \theta_{t} u+\nu_{t}+\left(\theta_{y} u+\nu_{y}\right) u+\theta\left(u^{2}+1\right)=\varepsilon_{1} a_{1}^{2}(\theta u+\nu) .
$$

Splitting the two equations in powers, we obtain the system of equations for functions $\theta, \nu$ :

$$
\theta_{t}=\nu_{y}, \quad \theta_{y}+\theta=0, \quad \theta_{t}+\nu_{y}=\varepsilon_{1} a_{1}^{2} \theta, \quad \nu_{t}+\theta=\varepsilon_{1} a_{1}^{2} \nu
$$

Solving these equations consequentially, we arrive to the contradictory condition $a_{1}^{4}=-1$.

Acting in a similar way, applying conditions (17)-(18), we have identified all others pairs of equivalent equations from Table III invariant under five- and four-dimensional algebras. In the Table IV we give the functions $f^{1}, f^{2}$ and $F^{1}, F^{2}$ describing that pairs as well as corresponding the additional equivalence transformations.

Finally, we exclude locally equivalent equations from Table III using Table IV and formulate the theorem.

Theorem 6 All possible equations of the form (1) admitting Lie algebras $A^{\mathrm{max}} \supset A^{\mathrm{ker}}$ of symmetries are reduced to one of the 14 "canonical" equations with functions given in Table $\mathrm{V}$ by an equivalence transformations from $G^{\text {equiv }}$ and the relevant additional equivalence transformations from Table IV. The maximal algebras of invariance of these "canonical" equations (1) are presented in the fourth column of Table $\mathrm{V}$. 
TABLE IV. Additional equivalence transformations.

\begin{tabular}{|c|c|c|c|c|c|}
\hline no. & $f^{1}(u)$ & $f^{2}(u)$ & $\begin{array}{c}\text { Equivalence } \\
\text { transformations }\end{array}$ & $F^{1}(\bar{u})$ & $F^{2}(\bar{u})$ \\
\hline 1 & $e^{u}$ & $\varepsilon_{1} e^{u}+\tilde{\varepsilon}_{2}$ & $\begin{array}{l}\bar{t}=t ; \bar{y}=-\varepsilon_{1} e^{-\varepsilon_{1} y} \\
\bar{x}=x ; \bar{u}=u-\varepsilon_{1} y\end{array}$ & $e^{\bar{u}}$ & $\tilde{\varepsilon}_{2}$ \\
\hline 2 & $\ln u$ & $\varepsilon_{1} u$ & $\begin{array}{l}\bar{t}=t ; \bar{y}=y-\frac{\varepsilon_{1}}{2} t^{2} \\
\bar{x}=x ; \bar{u}=e^{-\varepsilon_{1} t} u\end{array}$ & $\ln \bar{u}$ & 0 \\
\hline 3 & $\begin{array}{l}u^{m} \\
m \neq 0,1\end{array}$ & $\begin{array}{l}\varepsilon_{1} u^{m+1}+ \\
\tilde{\varepsilon}_{2} u\end{array}$ & $\begin{array}{l}\bar{t}=t ; \bar{y}=-\frac{\varepsilon_{1}}{m} e^{-\varepsilon_{1} m y} \\
\bar{x}=x ; \bar{u}=e^{-\varepsilon_{1} y} u\end{array}$ & $\bar{u}^{m}$ & $\tilde{\varepsilon}_{2} \bar{u}$ \\
\hline 4 & $u$ & $\varepsilon_{1}$ & $\begin{array}{l}\bar{t}=t ; \bar{y}=y-\frac{\varepsilon_{1}}{2} t^{2} \\
\bar{x}=x ; \bar{u}=u-\varepsilon_{1}\end{array}$ & $\bar{u}$ & 0 \\
\hline 5 & $u$ & $u^{2}$ & $\begin{array}{l}\bar{t}=t ; \bar{y}=-e^{-y} \\
\bar{x}=x ; \bar{u}=e^{-y} u\end{array}$ & $\bar{u}$ & 0 \\
\hline 6 & $u$ & $u^{2}-1$ & $\begin{array}{l}\bar{t}=2 t ; \bar{x}=\sqrt{2} x ; \bar{y}=-2 e^{t-y} \\
\bar{u}=e^{t-y} u-e^{t-y}\end{array}$ & $\bar{u}$ & $\bar{u}$ \\
\hline
\end{tabular}

Here $\varepsilon_{1}= \pm 1, \tilde{\varepsilon}_{2} \in\{-1,0,1\}$.

\section{EXACT SOLUTIONS}

\section{One-dimensional invariance subalgebras}

It is known that if a nonlinear differential equation with partial derivatives has nontrivial symmetry properties, it allows us to use differential operators of the invariance algebra for its symmetry reduction ${ }^{16,18}$.

We consider the use of the operators of the invariance algebra for symmetric reduction and the construction of exact solutions on the example of the nonlinear Kolmogorov equation (1) with the maximal six-dimensional invariance algebra. In Table III, we have three equations (cases 20, 21, 22) which have a six-dimensional invariance algebra.

Using the additional equivalence transformations from Table IV, we can reduce the cases 20 and 21 from Table III to the case 22, i.e., we arrive at the equation

$$
u_{t}-u_{x x}+u u_{y}=0
$$


TABLE V. Group classification of generalized nonlinear Kolmogorov type equations with respect to the additional equivalence transformations which do not belong to $G^{\text {equiv }}$

\begin{tabular}{llll}
\hline \hline No. & $f^{1}(u)$ & $f^{2}(u)$ & Generators from $A^{\text {max }} / A^{\text {ker }}$ \\
\hline 1 & $\forall \neq$ const & 0 & $t \partial_{t}+\frac{1}{2} x \partial_{x}+y \partial_{y}$ \\
2 & $e^{u}$ & $\varepsilon_{1} e^{m u}, m \neq 0,1$ & $t \partial_{t}+\frac{1}{2} x \partial_{x}+\frac{m-1}{m} y \partial_{y}-\frac{1}{m} \partial_{u}$ \\
3 & $e^{u}$ & $\varepsilon_{1}$ & $y \partial_{y}+\partial_{u}$ \\
4 & $e^{u}$ & 0 & $t \partial_{t}+\frac{1}{2} x \partial_{x}+y \partial_{y}, \quad y \partial_{y}+\partial_{u}$ \\
5 & $\ln u$ & $\varepsilon_{1} u^{m}, m \neq 1$ & $t \partial_{t}+\frac{1}{2} x \partial_{x}+\left(y-\frac{t}{m-1} \partial_{y}\right)-\frac{u}{m-1} \partial_{u}$ \\
6 & $\ln u$ & $\varepsilon_{1} u \ln u$ & $e^{\varepsilon_{1} t}\left(\partial_{y}+\varepsilon_{1} u \partial_{u}\right)$ \\
7 & $\ln u$ & $t \partial_{t}+\frac{1}{2} x \partial_{x}+y \partial_{y}, \quad t \partial_{y}+u \partial_{u}$ \\
8 & $u^{m} m \neq 0,1$ & $\varepsilon_{1} u^{n} n \neq m+1$ & $(n-1) t \partial_{t}+\frac{1}{2}(n-1) x \partial_{x}+(n-m-1) y \partial_{y}$ \\
9 & $u^{m} m \neq 0,1$ & 0 & $t \partial_{t}+\frac{1}{2} x \partial_{x}+y \partial_{y}, \quad y \partial_{y}+\frac{1}{m} u \partial_{u}$ \\
10 & $u$ & $\varepsilon_{1} e^{u}$ & $t \partial_{t}+\frac{1}{2} x \partial_{x}+(y-t) \partial_{y}-\partial_{u}$ \\
11 & $u$ & $\varepsilon_{1} u^{m} m \neq 0,1,2$ & $t \partial_{t}+\frac{1}{2} x \partial_{x}+\frac{m-2}{m-1} y \partial_{y}-\frac{u}{m-1} \partial_{u}$ \\
12 & $u$ & $e^{y}\left(\sin t \partial_{y}+(\cos t+u \sin t) \partial_{u}\right)$, \\
13 & $u$ & $u^{2}+1$ & $e^{y}\left(\cos t \partial_{y}-(\sin t-u \cos t) \partial_{u}\right)$ \\
\hline \hline & & $y \partial_{y}+u \partial_{u}, \quad e^{\varepsilon_{1} t}\left(\partial_{y}+\varepsilon_{1} \partial_{u}\right)$ \\
& & $t \partial_{t}+\frac{1}{2} x \partial_{x}+y \partial_{y}, \quad t \partial_{y}+\partial_{u}, \quad y \partial_{y}+u \partial_{u}$ \\
\hline
\end{tabular}

Here $\varepsilon_{1}= \pm 1$.

Let us note that the equation (47) are well investigated and that most of the exact solutions given below have been constructed before ${ }^{4,8}$.

Consider the equation

$$
u_{t}-u_{x x}-u u_{y}=0
$$

which occurs in many problems of financial mathematics ${ }^{19}$.

Applying the equivalence transformation

$$
t \rightarrow t, \quad x \rightarrow x, \quad y \rightarrow-y, \quad u \rightarrow u
$$

to equation (47) and to the operators of the invariance algebra from 22 of Table III we get the equation (48), which is invariant with respect to the six-dimensional invariance algebra 
whose operators have the form:

$$
\begin{gathered}
P_{0}=\partial_{t}, \quad P_{1}=\partial_{x}, \quad P_{2}=\partial_{y}, \quad D_{1}=t \partial_{t}+\frac{1}{2} x \partial_{x}+y \partial_{y} \\
D_{2}=y \partial_{y}+u \partial_{u}, \quad G=t \partial_{y}-\partial_{u}
\end{gathered}
$$

Consider the algebra with basis operators

$$
\begin{aligned}
& X_{1}=D_{1}-D_{2}=t \partial_{t}+\frac{1}{2} x \partial_{x}-u \partial_{u}, \\
& X_{2}=P_{0}=\partial_{t}, \quad X_{3}=D_{2}=y \partial_{y}+u \partial_{u}, \\
& X_{4}=P_{2}=\partial_{y}, \quad X_{5}=G=t \partial_{y}-\partial_{u}, \quad X_{6}=P_{1}=\partial_{x},
\end{aligned}
$$

which satisfy such non-zero commutation relations:

$$
\begin{gathered}
{\left[X_{1}, X_{2}\right]=-X_{2}, \quad\left[X_{1}, X_{5}\right]=X_{5}, \quad\left[X_{1}, X_{6}\right]=-\frac{1}{2} X_{6}} \\
{\left[X_{3}, X_{5}\right]=-X_{5}, \quad\left[X_{2}, X_{5}\right]=X_{4}, \quad\left[X_{3}, X_{4}\right]=-X_{4}}
\end{gathered}
$$

Further use of nontrivial symmetry properties of equation (48) requires a classification of subalgebras of the algebra. Using the well-known method of classifying subalgebras of a Lie algebra (see, for example, ${ }^{14,20}$ ), we classify all one-dimensional and two-dimensional Lie subalgebras of the algebra (49) up to the action of transformations from the group of its inner automorphisms to which there corresponds a nontrivial reduction of equation (48) to partial differential equations and ordinary differential equations, respectively. Thus, up to those action, one-dimensional subalgebras are exhausted by such algebras:

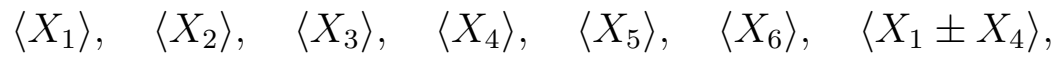

$$
\begin{aligned}
& \left\langle X_{1}+X_{3} \pm X_{5}\right\rangle, \quad\left\langle X_{1}+\alpha X_{3}\right\rangle, \quad\left\langle X_{2} \pm X_{3}\right\rangle, \quad\left\langle X_{3}+X_{6}\right\rangle, \\
& \left\langle X_{2} \pm X_{3} \pm X_{6}\right\rangle, \quad\left\langle X_{2} \pm X_{5}+\alpha X_{6}\right\rangle, \quad\left\langle X_{2}+X_{6}\right\rangle, \quad\left\langle X_{2} \pm X_{5}\right\rangle, \\
& \left\langle X_{5}+X_{6}\right\rangle, \quad\left\langle X_{4}+X_{6}\right\rangle, \quad(\alpha \neq 0) .
\end{aligned}
$$

Using these subalgebras, one can reduce equation (48) to two-dimensional partial differential equations. For example, consider the last operator from the list (50)

$$
\left\langle X_{4}+X_{6}\right\rangle=\partial_{x}+\partial_{y}
$$

Proceeding from the complete set of invariants of the operator (51)

$$
I_{1}=t, \quad I_{2}=y-x, \quad I_{3}=u,
$$


we seek an invariant solution in the form

$$
u=\varphi\left(\omega_{1}, \omega_{2}\right), \quad \omega_{1}=t, \quad \omega_{2}=y-x
$$

Substituting (52) into equation (48), we obtain the well-known Burgers equation

$$
\varphi_{\omega_{1}}-\varphi_{\omega_{2} \omega_{2}}-\varphi \varphi_{\omega_{2}}=0
$$

$\mathrm{In}^{19}$, the Cauchy problem for equation (48) is examined

$$
u_{t}-u_{x x}-u u_{y}=0, \quad u(0, x, y)=g(x, y) .
$$

If we require the solution of equation (48) to be invariant with respect to the operator (51), and the function was of the form $g=g\left(\omega_{2}\right)$ then the solution of the Cauchy problem

$$
\varphi_{\omega_{1}}-\varphi_{\omega_{2} \omega_{2}}-\varphi \varphi_{\omega_{2}}=0, \quad \varphi\left(0, \omega_{2}\right)=g\left(\omega_{2}\right)
$$

has the form (see, for example, ${ }^{22}$ )

$$
\varphi\left(\omega_{1}, \omega_{2}\right)=2 \frac{\partial}{\partial \omega_{2}} \ln G\left(\omega_{1}, \omega_{2}\right),
$$

where

$$
G\left(\omega_{1}, \omega_{2}\right)=\frac{1}{\sqrt{4 \pi \omega_{1}}} \int_{-\infty}^{\infty} \exp \left[-\frac{\left(\omega_{2}-\xi\right)^{2}}{4 \omega_{1}}-\frac{1}{2} \int_{0}^{\xi} g(\tau) d \tau\right] d \xi,
$$

and leads to the solution (52) of the Cauchy problem (53).

\section{Two-dimensional invariance subalgebras}

Let us employ the symmetries to reduce equation (48) to various ordinary differential equa-

tions. For this we classify all two-dimensional subalgebras of the algebra $L_{6}$ (49) up to the action of transformations of the group of its inner automorphisms. Wherein 49 twodimensional subalgebras are obtained. Among the obtained two-dimensional subalgebras, the most interesting from our point of view are the following, the corresponding ansatzes of which contain all the variables $t, x, y$ :

$$
\begin{aligned}
& \left\langle X_{1}, X_{5}\right\rangle, \quad\left\langle X_{1}, X_{3}\right\rangle, \quad\left\langle X_{1} \pm X_{4}, X_{5}\right\rangle, \quad\left\langle X_{1}+X_{3}, X_{2}+X_{4}\right\rangle, \\
& \left\langle X_{1}+2 X_{3}, X_{2}+X_{5}\right\rangle, \quad\left\langle X_{1}+\alpha X_{3}, X_{5}\right\rangle, \quad\left\langle X_{3}, X_{2}+X_{6}\right\rangle \\
& \left\langle X_{5}, X_{3}+X_{6}\right\rangle, \quad\left\langle X_{3}+X_{6}, X_{2}+\alpha X_{6}\right\rangle, \\
& \left\langle X_{2}+X_{5}+\beta X_{6}, X_{4}+\alpha X_{6}\right\rangle, \quad\left\langle X_{2}+X_{6}, X_{4}+\beta X_{6}\right\rangle, \\
& \left\langle X_{2}+X_{5}, X_{4}+\beta X_{6}\right\rangle, \quad\left\langle X_{5}, X_{4}+X_{6}\right\rangle, \quad\left\langle X_{5}+X_{6}, X_{1}+\frac{3}{2} X_{3}\right\rangle \\
& \left\langle X_{4}+X_{6}, X_{1}+\frac{1}{2} X_{3}\right\rangle, \quad\left\langle X_{5}+X_{6}, X_{4}+\alpha X_{6}\right\rangle \quad(\alpha \neq 0, \quad \beta>0) .
\end{aligned}
$$


We have considered some cases of the two-dimensional subalgebras (54) and obtained a symmetry reductions. For some of them we construct exact invariant solutions of equation (48). Let us give some examples.

Example 1: $\left\langle X_{4}+\alpha X_{6}, X_{5}+X_{6}\right\rangle$. The operators $X_{4}+\alpha X_{6}=\partial_{y}+\alpha \partial_{x}, X_{5}+X_{6}=$ $\partial_{x}+t \partial_{y}-\partial_{u}$ produces the similarity solutions

$$
u=\frac{x-\alpha y}{\alpha t-1}+\varphi(\omega), \quad \omega=t
$$

that reduces (48) to the ordinary differential equations $\varphi^{\prime}(\omega)=0$.

Solving the corresponding reduced equation, we obtain an invariant solution

$$
u=\frac{x-\alpha y}{\alpha t-1}+C
$$

where $C$ is an arbitrary constant.

$\mathrm{In}^{19}$ the exact solution of equation (48)

$$
u=\frac{x+y}{1-t},
$$

is considered. The solution (56) can be obtained from (55) with $C=0, \alpha=1$ and using the equivalence transformation $t \rightarrow t, x \rightarrow x, y \rightarrow-y, u \rightarrow-u$.

Example 2: $\left\langle X_{3}+X_{6}, X_{5}\right\rangle$. We obtain the similarity reduction

$$
u=-\frac{y}{t}+e^{x} f(\omega), \quad \omega=t,
$$

that reduces (48) to the differential equation

$$
\omega f^{\prime}+(1-\omega) f=0
$$

Solving the latter ordinary differential equation we obtain the similarity solution of equation (48)

$$
u=\frac{C e^{x+t}-y}{t}, \quad C=\text { const. }
$$

Example 3: $\left\langle X_{4}+X_{6}, X_{5}\right\rangle$. We obtain the similarity reduction

$$
u=\frac{x-y}{t}+f(\omega), \quad \omega=t ; \quad \omega f^{\prime}+f=0 .
$$

The reduced equations obtained obviously have exact solutions which lead to the above exact solution of equations (48)

$$
u=\frac{x-y+C}{t} .
$$


Example 4: $\left\langle X_{2}+X_{6}, X_{4}+\beta X_{6}\right\rangle, \beta>0$. We obtain the similarity reduction

$$
u=f(\omega), \quad \omega=\beta y+t-x ; \quad f^{\prime \prime}+(\beta f-1) f^{\prime}=0 .
$$

Substituting the solution of the reduced equation into the ansatz, we construct the exact solutions of equation (48):

$$
\begin{gathered}
u=\frac{2}{\beta\left(\beta y+t-x+C_{1}\right)}+\frac{1}{\beta} ; \\
u=\frac{\sqrt{-1-2 \beta C}}{\beta}+\tan \left(-\frac{\sqrt{-1-2 \beta C}}{\beta}(\beta y+t-x)+C_{1}\right)+\frac{1}{\beta}, \quad 2 \beta C+1<0 ; \\
u=\frac{-2 \sqrt{1+2 \beta C}}{\beta\left(C_{1} e^{-\sqrt{1+2 \beta C)}(\beta y+t-x)}-1\right)}+\frac{1-\sqrt{1+2 \beta C}}{\beta}, \quad 2 \beta C+1>0, \quad C, C_{1}=\text { const. }
\end{gathered}
$$

Example 5: $\left\langle X_{3}, X_{2}+X_{6}\right\rangle$. We obtain the similarity reduction

$$
u=y f(\omega), \quad \omega=t-x ; \quad f^{\prime \prime}-f^{\prime}+f^{2}=0,
$$

which in turn reduces to the Emden-Fowler equation, whose solutions are known for a number of parameter values ${ }^{12,21}$.

\section{CONCLUSIONS}

In this paper, the group classification in the class of nonlinear Kolmogorov equations (1) is presented completely. The main results on classification are collected in Table III, where we list inequivalent cases of extensions with the corresponding Lie invariance algebras. The list contains 22 equations and cannot be shortened by local substitution from group of equivalence transformations. In Table IV we write down all the additional equivalence transformations, reducing some equations from our classification to others of simpler forms. We have proved that exactly 14 equations in Table $\mathrm{V}$ are reduced to other equations listed by the additional equivalence transformations. For equations which have the maximal symmetry properties six-dimensional invariance algebra we can reduce these equations to equation (48) using the set additional equivalence transformations. Using the well-known method of classifying subalgebras of a Lie algebra, we classify all one-dimensional and two-dimensional Lie subalgebras of an algebra which corresponds a nontrivial reduction of equation (48) to partial differential equations and ordinary differential equations respectively. 


\section{REFERENCES}

${ }^{1}$ Akhatov, I. S., Gazizov, R. K., and Ibragimov, N. K., "Nonlocal symmetries. heuristic approach," J. Soviet Mathematics 55(1), 1401-50 (1991).

${ }^{2}$ Bluman, G. W. and Kumei, S., Symmetries and Differential Equations (Springer-Verlag, 1989).

${ }^{3}$ Cherniha, R., Serov, M., and Rassokha, I., "Lie symmetries and form-preserving transformations of reaction diffusion convection equations," J. Math. Anal. Appl. 342, 1363-79 (2008).

${ }^{4}$ Demetriou, E., Christou, M. A., and Sophocleous, C., "On the classification of similarity solutions of a two-dimensional diffusion-advection equation," J. Appl. Math. Comp. 187, $1333-50(2007)$.

${ }^{5}$ Demetriou, E., Ivanova, N., and Sophocleous, C., "Group analysis of $(2+1)-$ and $(3+1)$ dimensional diffusion-convection equations," J. Math. Anal. Appl. 348, 55-65 (2008).

${ }^{6}$ Dorodnitsyn, V. A., "On invariant solutions of the equation of non-linear heat conduction with a source," U.S.S.R. Comput. Math. Math. Phys. 22, 115-22 (1982).

${ }^{7}$ Dorodnitsyn, V. A., Knyazeva, I. V., and Svirschevsky, S. R., "Group properties of the heat equation with a source in two-dimensional and three-dimensional cases," Diferential equations 19, 1215-23 (1983).

${ }^{8}$ Elwakil, S. A., Zahran, M., and Sabry, R., "Group classification and symmetry reduction of a $(2+1)$ dimensional diffusion-advection equation," J. Applied Math. Phys. 56, 986-99 (2005).

${ }^{9}$ Huang, D. and Ivanova, N. M., "Algorithmic framework for group analysis of differential equations and its application to generalized zakharov-kuznetsov equations," J. Differential Equations 260, 2354-82 (2016).

${ }^{10}$ Ibragimov, N. H., ed., "Crc handbook of Lie group analysis of differential equations," (CRC Press, 1994).

${ }^{11}$ Ibragimov, N. H., ed., "Crc handbook of Lie group analysis of differential equations," (CRC Press, 1995) pp. 291-328.

${ }^{12}$ Kamke, E., ed., "Differentialgleichungen: Lösungsmethoden und lösungen, I, gewöhnliche differentialgleichungen," (B. G. Teubner, Leipzig, 1977). 
${ }^{13}$ Kingston, J. G. and Sophocleous, C., "On form-preserving point transformations of partial differential equations," J. Phys. A: Math. Gen. 31, 1597-619 (1998).

${ }^{14}$ Lahno, V. I., Spichak, S. V., and Stogniy, V. I., eds., "Symmetry Analysis of Evolution Type Equations," (Kyiv; Institute of Mathematics of NAS of Ukraine, 2002 (in Ukrainian)).

${ }^{15}$ Nikititin, A. G. and Popovych, R., "Group classification nonlinear Schrodinger equations," Ukr. Math. J. 53, 1255-65 (2001).

${ }^{16}$ Olver, P. J., Application of Lie Groups to Differential Equations (Springer, 1986).

${ }^{17}$ Ovsiannikov, L. V., "Group properties of nonlinear heat equation," Dokl. AN SSSR 125, 492-5 (1959).

${ }^{18}$ Ovsiannikov, L. V., Group Analysis of Differential Equations (Acad. Press, 1982).

${ }^{19}$ Pascucci, A. and Polidoro, S., "On the Cauchy problem for a nonlinear Kolmogorov equation," SIAM J. Math. Anal. 35, 579-95 (2003).

${ }^{20}$ Patera, J. and Winternitz, P., "Continuous subgroups of the fundamental groups of physics. I. general methods and the poincare group," J. Math. Phys. 16, 1597-1624 (1975).

${ }^{21}$ Polyanin, A. D. and Zaitsev, V. F., eds., "Handbook of exact solutions for ordinary differential equations," (Chapman \& Hall/CRC, Boca Raton, FL, 2003).

${ }^{22}$ Polyanin, A. D. and Zaitsev, V. F., eds., "Handbook of nonlinear partial differential equations," (Chapman \& Hall/CRC, Boca Raton, FL, 2004).

${ }^{23}$ Popovych, R. O. and Ivanova, N. M., "New results on group classification of nonlinear diffusion-convection equations," J. Phys. A: Math. Gen. 37, 7547-65 (2004).

${ }^{24}$ Shtelen, W. M. and Stogny, V. I., "Symmetry properties of one- and two-dimensional Fokker-Planck equations," J. Phys. A: Math. Gen. 22, 539-43 (1989).

${ }^{25}$ Spichak, S. V. and Stogniy, V. I., "Symmetry classification and exact solutions of the Kramers equation," J. Math. Phys. 39, 3505-10 (1998).

${ }^{26}$ Vasilenko, O. F. and Yehorchenko, I. A., "Group classification of multidimensional nonlinear wave equations," Proc. Inst. Mathematics of NAS of Ukraine 36, 63 (2001).

${ }^{27}$ Winternitz, P. and Gazeau, J., "Allowed transformations and symmetry classes of variable coefficient Korteweg-de Vries equations," Phys. Lett. A 167, 246-50 (1992). 